\title{
Interview with Dr Richard Budgett, medical and scientific director for the IOC
}

\author{
ELITE SPORTS \\ INTERVIEW \\ SPORTS AND SOCIETY \\ SPORTS AND YOUTH \\ SPORTS MEDICINE / TEAM
}

MANAGEMENT

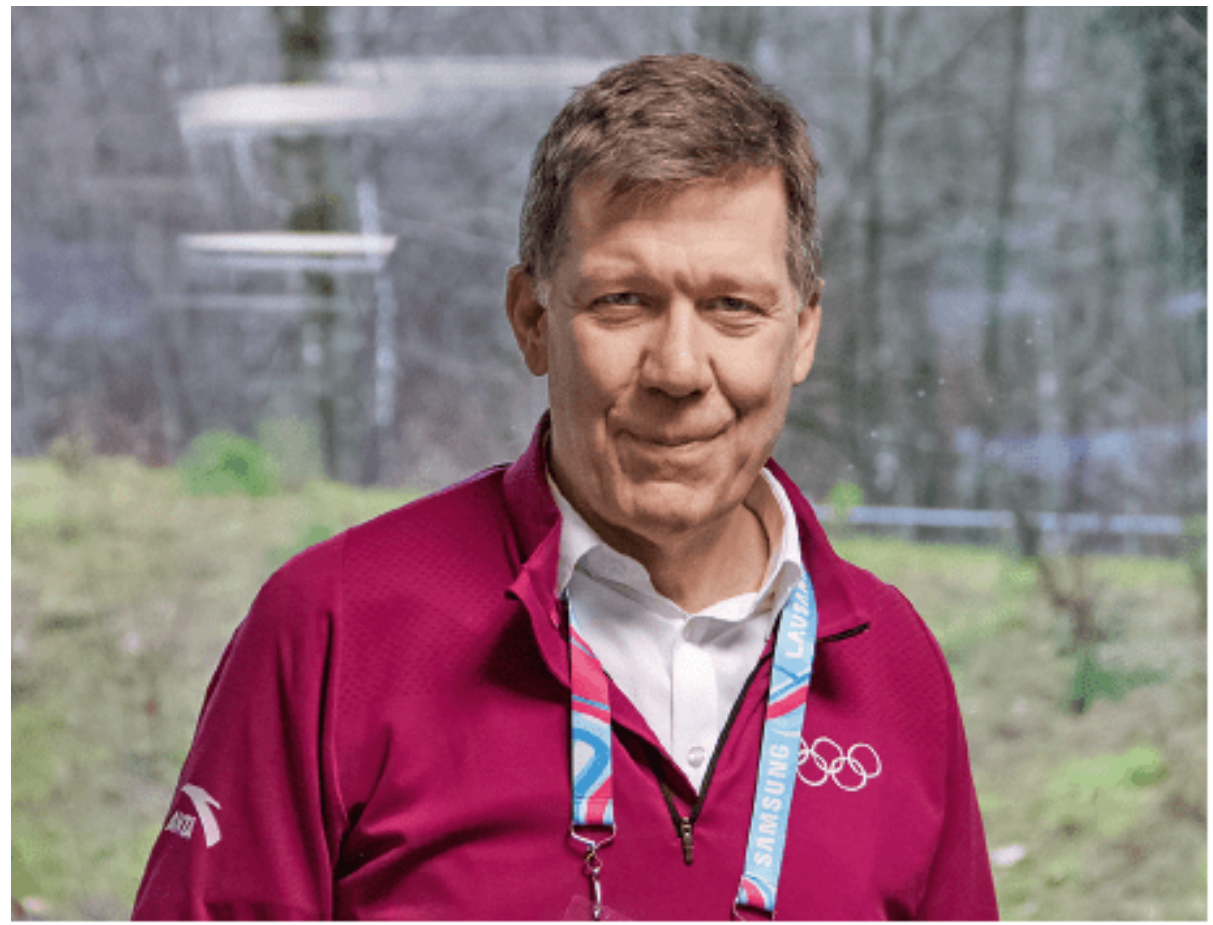

This interview was conducted by Dr Boris Gojanovic after the Lausanne 2020

\section{Youth Olympic Games.}

\section{Richard, can you please introduce yourself, as a medical doctor and an athlete.}

I am the medical and scientific director for the IOC and I started off in life as a rower. As an athlete, I had the privilege to compete in the 1984 Olympic Games in Los Angeles, and I won a gold medal there. Then I went to complete my medical training and became a team doctor involved with the rowing team (not surprisingly), but also with the British bobsleigh team. Then I became the chief medical officer for the Great Britain team for 16 years, and then in the lead up to 2012 I became the chief medical officer for the London 2012 Olympics. Immediately after that I came to the IOC, so I have been in Lausanne for the last 7 years.

Please describe your role at IOC.

As the medical and scientific director, I am responsible for making sure that the medical and scientific 
commission and the department fulfil their mission. The main mission is to protect the health of athletes. In fact, it is to protect the health of everyone involved with the Games but our priority is for the athletes. So we have to get it right at the Games, but we also have a major program of education and of research, and our priority is prevention of injury and illness in athletes. We don't just try and prevent injuries, which is hugely important, but also aspects of illness and wellness such as prevention of harassment and abuse in sport, mental health, things like that.

\section{What is the mission and goal of the Youth Olympic Games (YOG), as viewed from the Medical Commission?}

The Youth Olympic Games in Lausanne were very successful, and it is difficult to believe it started 3 months ago. It is the same mission as for the commission and the department, which is to protect the health of the athletes. We do that, obviously not on our own, but really relying hugely on the organizing comity, on the team doctors, the National Olympic Committee (NOC) doctors and on the international federation doctors. But really in protecting the health of the Youth Olympic Games, it is all about education coming first and disseminating that education. I suppose the YOG is an opportunity to try new initiatives and that makes it often very exciting.

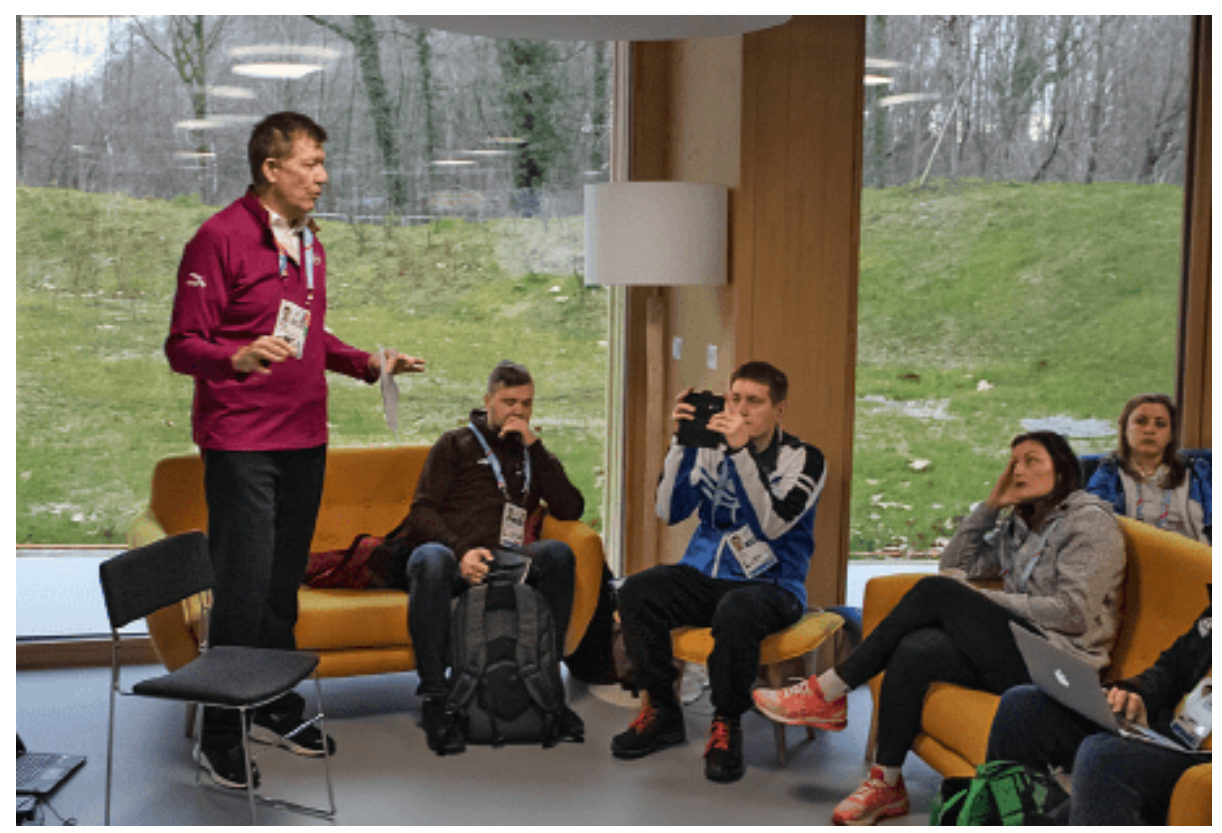

Figure 1: Teaching at the educational session at the medical clinic.

\section{What did Lausanne 2020 represent for you and the IOC in general?}

Well, it is easiest for me to talk from my own personal experience; this was a unique chance to hold an Olympics on our own ground, in the Capitale Olympique. There were challenges because we would carry on with normal life, they were loads of distractions to do with the Tokyo Games or with other priorities for the IOC that you could not get away from. Normally when you are at a YOG, you are in that Olympic bubble, isolated from the rest of the world and totally focused on the Olympic Games you are at. But I suppose to us, the YOG of Lausanne represented bringing it home, to the home of Olympism, and really the 
excitement of having the Games here. For me there was a huge confidence, because I have been here for more than 7 years, I know the medical system, so I could be relaxed and confident about the medical support that was going to be available.

\section{What do you think of the Health for Performance concept in general?}

The Health for Performance is a really good concept, in practice it is exactly what our priorities are as well. It fits with the mission of the IOC medical \& scientific commission and department, which is protecting the health of athletes and health coming first. So it really encompasses everything as it did, from prevention of harassment and abuse in sport through to injury prevention.

\section{Were you able to visit some of the activities at the Vortex H4P area? Which ones, and what was your experience like?}

It was very nicely set up in the Vortex, which was the Youth Olympic Village, with a number of activities and stations for the athletes to take part in. I visited on several occasions and I saw the athletes take part in assessments, looking at their flexibility, agility, skills, with advice on mental health, activities around what does abuse mean in sport and so, obviously, how to prevent it. And then very memorably concussion and, with the so called Deep impact virtual reality. I did take part in that myself and was able to experience just how effective it was, what good learning too it was to make athletes and their entourage realise how important it is to take the appropriate measures after a concussion before resuming participation.

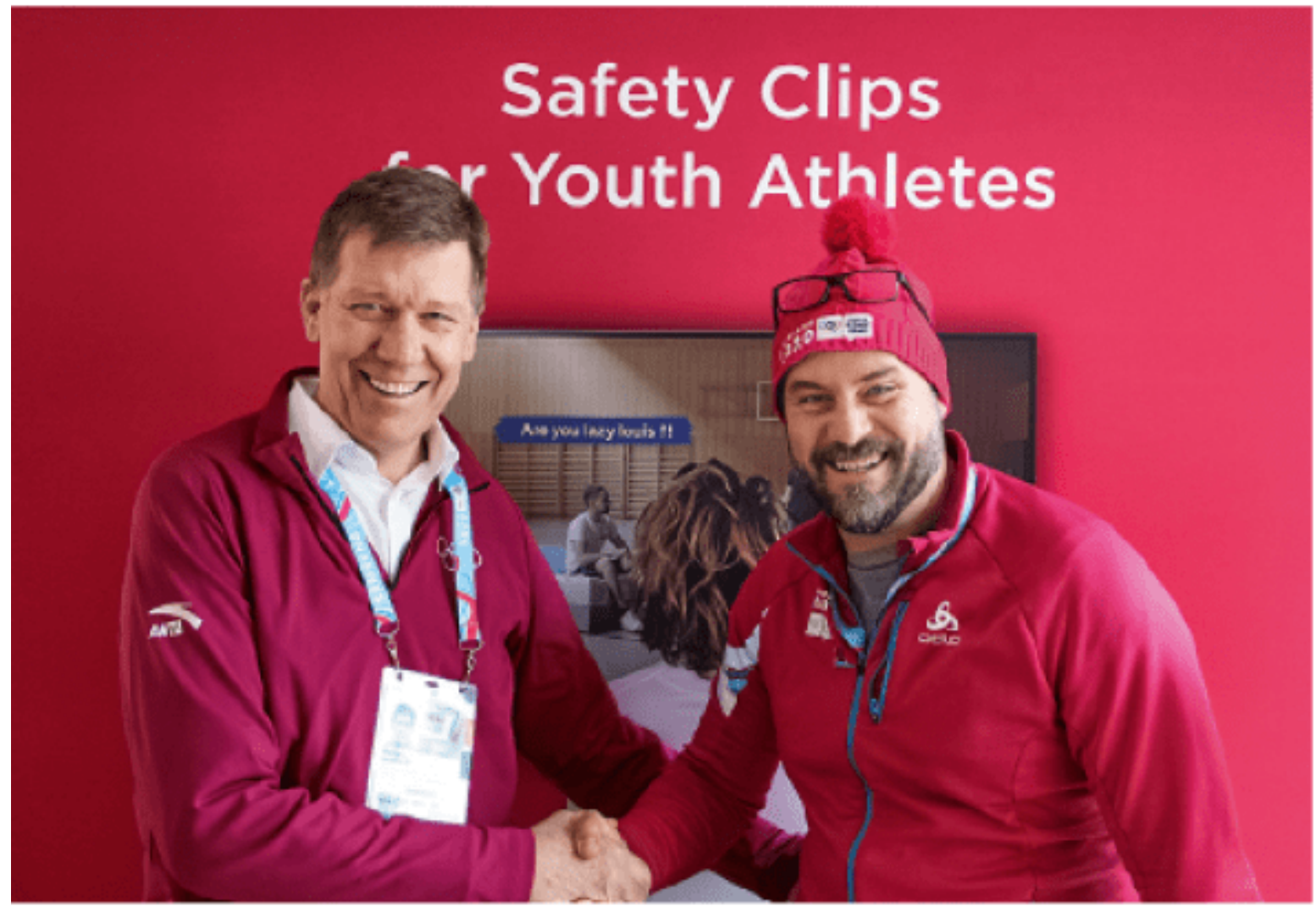

Figure 2: Richard Budgett with the Medical clinic director, Dr Stéphane Tercier. 


\section{level of competition?}

I think there are challenges with any exercise. We know that sport and exercise is good for health, but one of the reason that sport medicine exists is that if you do any activity there is a risk of injury. So really, what we need is research and education. We have something called the Olympic Movement Medical Code and this is very important for empowering doctors, physiotherapist, the whole medical support team and the athlete's entourage to support the athlete in the right way. So the athlete can have the right access, the appropriate level of confidentiality and they are all entitled to health care. But that is around actually treating and managing injury or illness when they happen. Clearly prevention has been our priority has been for the IOC in the last 10 years. If we can have evidence based prevention then we can promote healthy sports participation.

\section{What are the challenges that you are facing to best protect the health of the athletes?}

One of the issues is that we don't know all the answers. It is clear in this current time of Covid-19 that medicine and science don't know all the answers. We learn lots and we continue to push to learn lots. So one of the challenges is, where we do know the answers - and there is a lot of various sport injury where we do have really good evidence - is translating that into practice. So the adoption of injury prevention strategies which we know will reduce injuries by $50 \%$ in many sports, it is not universal because of the difficulties of behavioural change, people's routine, it's difficult to change people's ideas on how to do sport, how to train, etc. The other important challenge in the Olympic context is to get the NOC doctor, the team doctors, the organising committee medical staff, the international federation medical staff and the IOC all working together. You have got 4 different bodies looking after athletes in the context of the Olympic Games. The coordination of our prevention and management of illness and injury is really important. And I think social media and modern communication have made a big difference. Around Covid-19 there is a hashtag: \#stayhealthy, \#staystrong, \#stayactive, and that has had a big impact. We can have a similar impact, I think, with injury and illness prevention.

\section{What do you wish to tell to young sports medicine doctors that dream of working at the highest level of competition?}

It's funny because it seems like yesterday when I was in that position and trying to get in sport. Obviously it helps if you are taking part in sport, but it is absolutely not a necessity. If you are infused about treating and helping athletes to stay healthy so they can perform, then sports and exercise medicine is for you. And it is a matter of taking every opportunity, even volunteering, working with the multidisciplinary team in the widest sense, that is not just the physiotherapist, osteopath or chiropractor, but also the strength and conditioning experts, the trainers, the coaches, so you are really working together and getting to understand and have a real knowledge of a whole variety of the sports, not just the few that you might be interested in, but all the sports. And every time as a young doctor you see an athlete from a new sport, take a little bit of extra time to find out about that sport, to understand the way they speak, the terms they have, because that will help you when you see a subsequent athlete from the same sport. 


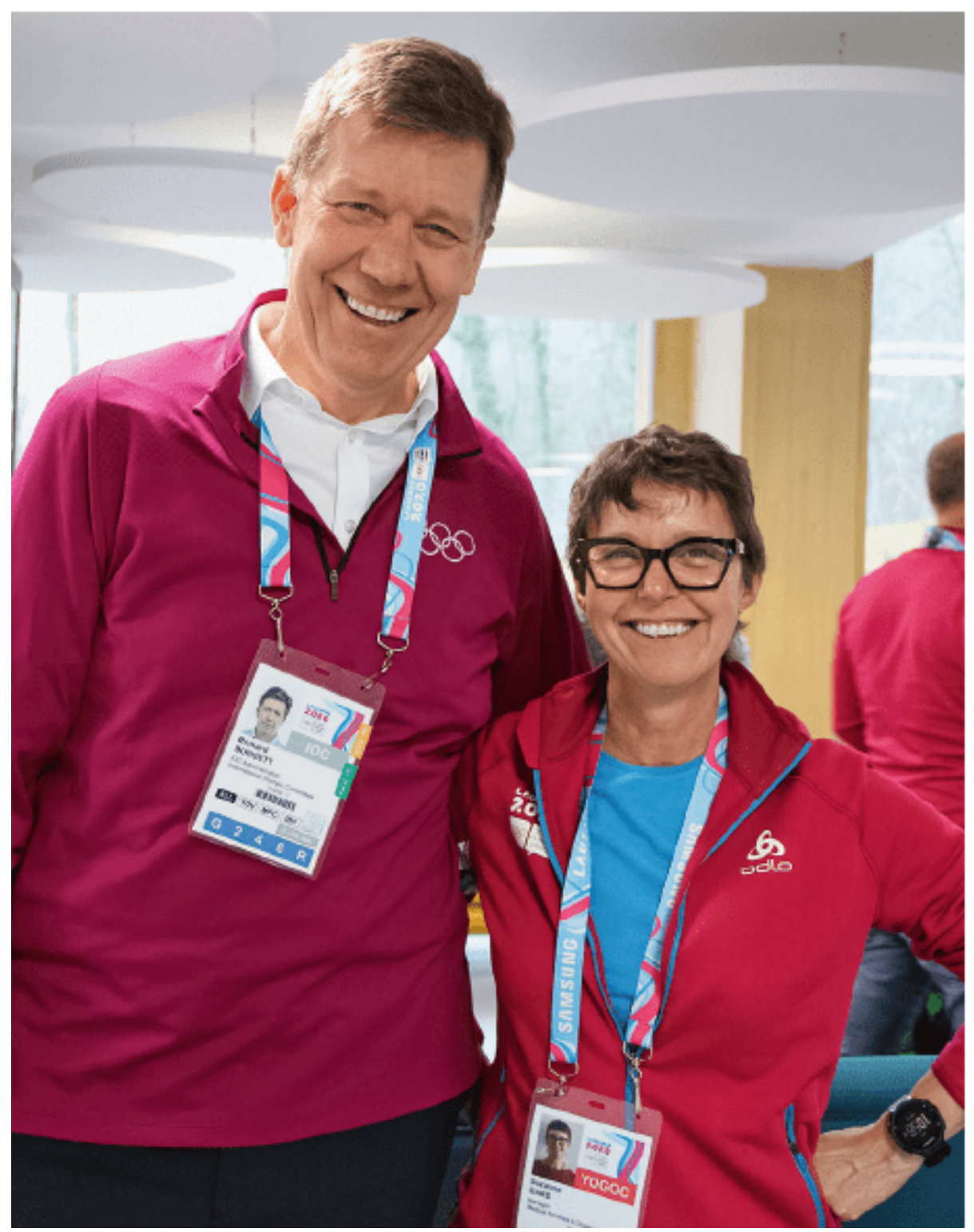

Figure 3: Richard Budgett with Suzanne Gard, the educational program coordinator.

\section{What is the future of prevention and educational programs for YOGs (and OGs)?}

The future as we have seen in the last month or two, is digital. It is more use or the internet, the apps and programs. We have got some of it already, and it is compulsory for all doctors who have registered for the Olympic Games to have completed what is called a "sports physician toolkit", which is part of the elearning that the World Antidoping Agency (WADA) has. They put that together with the IOC and we have plans to put together further compulsory modules so that doctors understand their responsibilities and have a basic knowledge when they come into the Olympic Games. I think we will broaden that out. We have already got e-learning within the medical scientific department of the IOC, which has been going on for 10 years, with diplomas in sport nutrition, in sport and exercise medicine and physiotherapy, and in drugs in sport. There is an extensive e-learning program and I think this will get tailored more to the Olympic Games. It will become so that you can take different modules, the ones that interest you at the 
time so that we can make sure that everybody who is looking after the athletes have the latest evidence based information.

\section{Any particular area that you feel needs particular attention in the coming years?}

All aspects are important. I would say, and I can say this without embarrassment because it started before I came to the IOC 7 years ago, that for the last 10 years, the IOC has really lead the way in injury prevention. Something that really wasn't spoken about much is now central to much of what we do in sport and exercise medicine. I think we can build on that and think about illness, which is now included as prevention of illness, but also really important areas like mental health, which the IOC is tackling on a multidisciplinary basis. It is about how to put all the fantastic information and knowledge we have in practice in sports - that is about behavioural change - so we can move all the theoretical injury and illness prevention into reality across athletes in every continent.

\section{IOC worked with local medical and allied healthcare professionals for Lausanne2020, what was your experience like?}

Despite my handicap with the language issue - unfortunately despite 7 years in Lausanne in a French speaking country, I am still really struggling — I was still able to really benefit from a fantastic organizing committee. The medical department and the organizing committee were superb. I knew before we even started of their great experience and expertise, but their enthusiasm was really impressive. They were really professional and it was a real pleasure to work with the organizing committee and I know it was the same for all the team doctors and IOC doctors who were there as well. As a medical fraternity, we had a great experience. One of the fantastic initiatives was the education that was done and organized by the organizing committee in the clinic at the Youth Olympic Village. That was in a unique format of having short 30 minute presentations and discussions twice a day, so that team doctors, physiotherapists and organizing committee medical staff could turn up when they were able to, and take part in that. It was good team building as well as being important information delivered and shared.

\section{Can you share one special moment from Lausanne 2020?}

That is difficult. Obviously, there were a lot of special moments. Both in the occasions I got out to see athletes competing and then with my friends and colleagues in the organizing committee with our small IOC medical commission Games group that was there. I suppose seeing the medical staff and volunteers engaging with each other in those educational sessions in the clinic at the Vortex, and also seeing them engaging with athletes was very special. I was in the clinic on one occasion when the mother of quite a badly injured athlete came in with her daughter in a wheelchair, and was just full of thanks for everybody. I know that meant a lot for the people who were there, and I had a sense of reflected thanks as well. It just showed that all the work to put the systems in place to make sure the athletes were supported in the best possible way was effective and was really appreciated. So that was a special moment. 


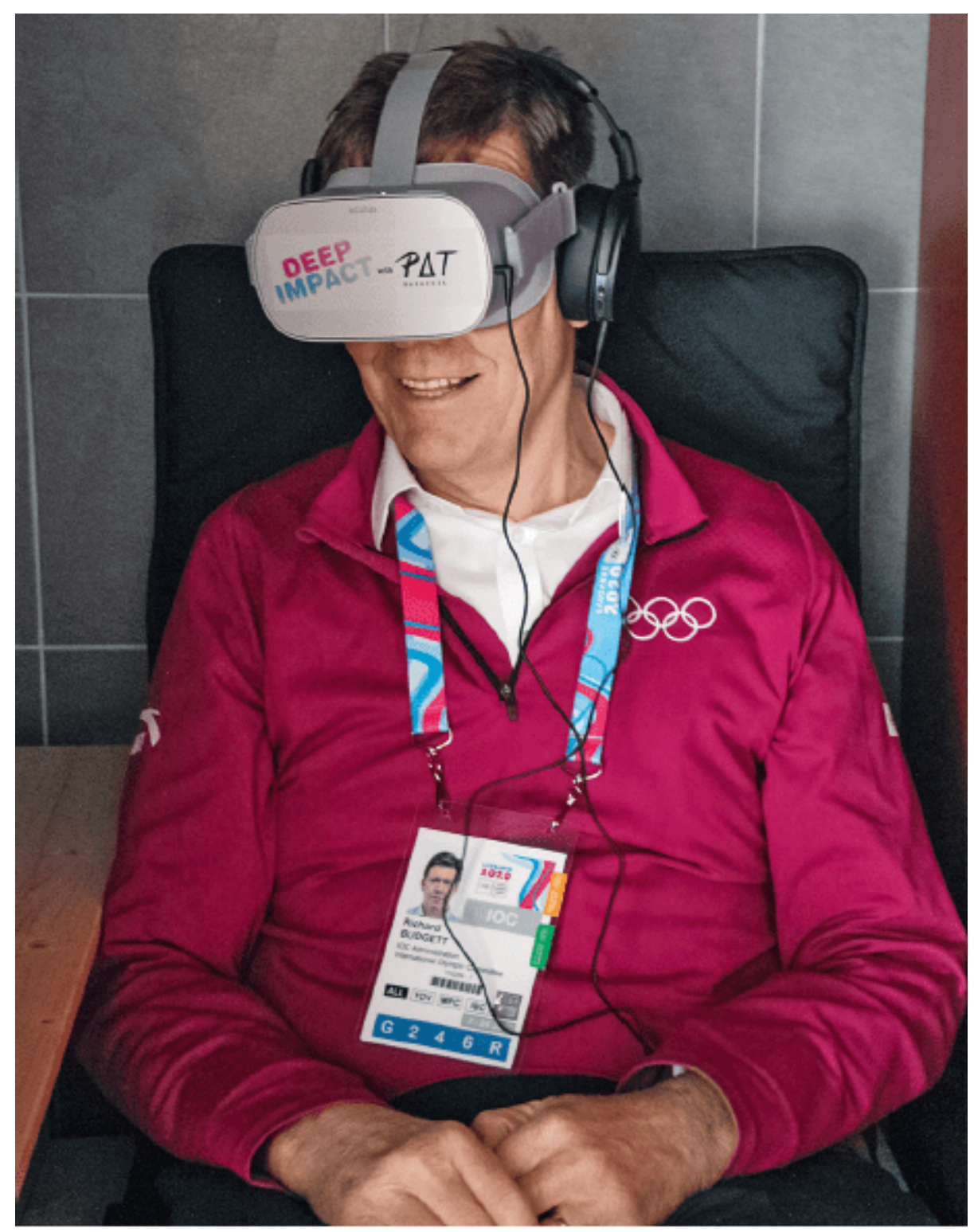

Figure 4: Dr Budgett experiencing the Deep Impact concussion virtual reality module.

Thank you very much for your time, Dr Budgett, I am sure our readers will really appreciate your statements and considerations around the YOG and the health of the athletes. 